\title{
Medication safety in pregnancy - Results from the MoBa study
}

\author{
Hedvig Nordeng ${ }^{1,2}$, Eivind Ystrom ${ }^{1}$ and Malin Eberhard-Gran ${ }^{1}$ \\ 1) Division of Mental Health, National Institute of Public Health \\ 2) Pharmacoepidemiology and Drug Safety Research Group, School of Pharmacy, University of Oslo \\ Correspondence: Hedvig Nordeng, School of Pharmacy, University of Oslo, PB 1068 Blindern, NO-0316 Oslo, Norway \\ E-mail: h.m.e.nordeng@farmasi.uio.no Telephone: +4722856604
}

\begin{abstract}
SUMMARY
This article summarizes the results of several of our studies on medication safety in pregnancy based on the Norwegian Mother and Child Cohort Study (MoBa). Medications investigated include antidepressants, NSAIDs, codeine, triptans, paracetamol and certain herbals. A major advantage of these studies is that MoBa has information on prescribed medications, over-the-counter medications and herbal medications. Moreover, MoBa enables the possibility of including a disease comparison group, and long-term follow-up into childhood. The size of MoBa enables designs like the sibling-design, which offers important advantages over studies comparing unrelated individuals. The possibility of linking MoBa to nationwide registries like the NorPD and the National Patient Registry enables validation of medication exposures and childhood diagnosis. Pharmacoepidemiological studies are vital to our understanding of the safety of medications in pregnancy, but great care must be taken in the analysis and interpretation of observational data to avoid problems of confounding and bias.
\end{abstract}

This is an open access article distributed under the Creative Commons Attribution Licence, which permits unrestricted use, distribution, and reproduction in any medium, provided the original work is properly cited.

\section{INTRODUCTION}

Because drugs may cause injury to the unborn child, we need to know which drugs are safe to use during pregnancy and which are not. This lesson was learned in the 1960s and 1970s when almost 10,000 children were born with malformed extremities after exposure to thalidomide during pregnancy. Today, up to eight out of ten women use one or more medications during pregnancy, many of them without sound information about safety in pregnancy (1). Almost 6 out of 10 women in Norway filled at least one prescription medication during pregnancy according to the Norwegian Prescription Registry (NorPD) (2). Though caution in the use of psychiatric medication is recommended, studies show a marked increase in use of antidepressants in pregnancy in the last ten years, with the most recent prevalence of use ranging from to $3-4 \%$ in Europe and Australia up to $8.3 \%$ in North America (1). Over half of all pregnant women use analgesics during pregnancy, most commonly over-the-counter paracetamol $(1,3)$.

Avoiding all drugs in pregnancy is unrealistic, and may even be dangerous. Untreated severe mental illnesses, epilepsy, diabetes, and infections in pregnancy are conditions in which the underlying illness itself may be harmful to the fetus, and thus require treatment. On the other hand, superfluous use of drugs may also occur during pregnancy and have potential negative consequences to the health of the woman and the unborn child.

In the general population, our knowledge of medication safety is based on clinical trials. However, pregnant women are routinely excluded from these studies due to uncertainty about the effects of medication on fetal development. Therefore, in practice, pharmacoepidemiological studies provide the best way of evaluating whether an exposure during pregnancy has adverse effects on the developing fetus (4).

\section{AIM}

The aim of this article is to summarize the results of several of our studies on medication safety in pregnancy based on The Norwegian Mother and Child Cohort Study (MoBa). Moreover, we will discuss some of the challenges in interpreting results from pharmacoepidemiological studies and criteria for causation in teratology.

\section{OVERVIEW OF MOBA "MEDiCATION SAFETY in PregnanCY" sub-STUdies}

\section{Antidepressants}

Two of our MoBa sub-studies focused on the safety of antidepressants, which is probably the most debated medication group with respect to safety in pregnancy. Results from previous medication safety studies have been conflicting (5). As many women will have to continue their antidepressant therapy during pregnancy, it is essential to establish their safety and determine whether there are differences in risk between different groups of antidepressants. Psychiatric illnesses are common among women in reproductive age, affecting up to $20 \%$ of all pregnant women (6), and untreated severe mental illnesses may in itself be harmful to the fetus (5).

In the first study, 699 of the $63,395 \mathrm{MoBa}$ women (1.1\%) reported using antidepressants during pregnancy, most frequently SSRIs (0.9\%) (7). Exposure to SSRIs during the first trimester was not associated with increased risk of overall congenital malformations (adjusted odds ratio (OR) 1.22; 95\% confidence interval (CI) 0.81-1.84) or cardiovascular malformations (adjusted OR 1.51; 95\% CI 0.67-3.43). Exposure to antidepressants during pregnancy was not associated with increased risk of low birth weight (adjusted OR 0.62; 95\% CI 0.33-1.16). 
In crude analyses, exposure to antidepressants was associated with an increased risk of preterm birth (crude OR 1.46; 95\% CI 1.04-2.04); however, after adjustment for depression and socio-demographic characteristics no increased risk of preterm birth was identified (adjusted OR 1.21; 95\% CI 0.87-1.69). In fact, having symptoms of depression in pregnancy was in itself significantly associated with preterm birth (adjusted OR 1.13; 95\% CI 1.03-1.25).

These findings are reassuring with respect to antidepressant use during pregnancy and illustrate the importance of adjustment for the underlying illness in pharmacoepidemiological studies. Without adjustments for level of maternal depression and various socio-demographic and lifestyle factors, antidepressant use during pregnancy would wrongly have been associated with an increased risk of preterm birth.

The background for the second study was lack of knowledge about risks of vaginal bleeding and postpartum hemorrhage after use of antidepressants in pregnancy (8). An increase in the risk of gastrointestinal bleeding and bruising had been reported in nonpregnant subjects after use of SSRI-antidepressants, possibly due to an effect on serotonin in blood platelets and thereby on hemostasis. Analyses were performed according to type of antidepressant, trimester of exposure, amount of bleeding, number of bleeding episodes and timing of bleeding in pregnancy/post-partum. No increased risk of bleeding complications was identified after use of antidepressants in pregnancy. However, the disease comparison group (non-medicated women with symptoms of depression) had a moderately increased risk of vaginal bleeding in early (adjusted OR 1.28; 95\% CI 1.07-1.55) and midpregnancy (adjusted OR 1.24; 95\% CI 1.00-1.55). These women had more severe symptoms of depression than their medicated counterparts, suggesting a potential involvement of the underlying maternal depression in vaginal bleeding outcomes that should be explored in future studies.

\section{Analgesics}

We have published four articles on safety of analgesics based on data from the MoBa cohort, including two on NSAIDs $(9,10)$, one on codeine (11) and one on triptans (12). Based on animal and prior epidemiological studies indicating a possible link between COX-1 inhibition and cardiac, midline and diaphragm defects (13), we wished to explore the association between exposure to NSAIDs in the first 12 weeks of gestation and these malformations. We found that despite being contraindicated in the first trimester, over 3000 women in $\mathrm{MoBa}(4.5 \%)$ reported to have used NSAIDs in early pregnancy (9). No significant increased risk of congenital heart defects or orofacial clefts was detected, although there was a non-significant increased risk of septal defects after exposure to multiple NSAIDs in the first 12 weeks of gestation (2 exposed cases; crude odds ratio 3.9, 95\% CI 0.9-15.7) (9). Previously Ofori et al. reported a similar increased risk of septal defects after exposure to NSAID in the first trimester (21 exposed cases; adjusted OR 3.3; 95\% CI 1.9-6.0) (14). Consequently, this finding should be explored in future studies with sufficient power to assess the risk of septal defects after exposure to specific NSAID during the first trimester of pregnancy.

As a continuation of this study, we performed a second NSAID study including additional pregnancy outcomes like low birth weight, premature delivery, maternal hemorrhage and referral to a specialist for asthmatic symptoms in the child at 18 months (10). The last two outcomes were selected as NSAIDs inhibit the blood clotting function of platelets by inhibiting prostaglandin synthesis, and may worsen symptoms of asthma in sensitive patients (13).

In total, $6511(7.2 \%)$ reported using one or several of the NSAIDs, most commonly ibuprofen $(n=5325$, $5.9 \%$ ) (10). There was a borderline significant association between ibuprofen use during the first trimester and structural heart defects in the 18-month-old child ( $1.5 \%$ vs. $1.2 \%$ among non-exposed controls, adjusted OR 1.2; 95\% CI 1.0-1.6) (10). Second trimester exposure to ibuprofen (adjusted OR 1.7; 95\% CI 1.3-2.3) or diclophenac (adjusted OR 3.1; 95\% CI 1.1-9.0) was significantly associated with low birth weight. Moreover, infants exposed to ibuprofen in the second trimester (adjusted OR 1.5; 95\% CI 1.2-1.9) or in the third trimester (adjusted OR 1.5; 95\% CI 1.1-2.1) had an increased risk of having asthmatic symptoms at 18 months of age compared to non-exposed infants. Finally, several of the NSAIDs were associated with an increased risk of vaginal bleeding and post-partum hemorrhage (10).

These results support current pharmacotherapeutic guidelines that contraindicate NSAIDs in the first and third trimester, and recommend caution with use in the second trimester of pregnancy (13).

For women in need of stronger analgesics than paracetamol or NSAIDs, the first line choice is codeine in a fixed combination with paracetamol $(30 \mathrm{mg}$ codeine + paracetamol 400-500 mg). Leading literature sources on drug safety in pregnancy consider that sporadic use of codeine is safe in pregnancy $(13,15)$, but surprisingly few studies have been conducted on codeine use in pregnancy in therapeutic doses in a population-based sample. In our MoBa sub-study, 1449 women (2.1\%) used codeine during pregnancy $(98 \%$ in a fixed combination of codeine/paracetamol), of whom 627 (0.9\%) used it in the first trimester $(11,16)$. No significant difference in the major congenital malformation rate was found between infants exposed to codeine in the first trimester $(2.6 \%)$ and the unexposed group (3.0\%) (adjusted OR 0.8; 95\% CI 0.5-1.4) (11,16). Of note, codeine use was associated with both planned and acute caesarean delivery, probably due to the woman's underlying pain condition. These results are reassuring with respect to congenital malformations.

Our interest in treatment of pain in pregnancy led us 
to focus on a specific group of women often in need of analgesics, namely women with migraine. Migraine affects more than $15 \%$ of women, and although migraine symptoms will improve for many of them during pregnancy, pharmacotherapy is essential for some pregnant women (13). Untreated or inadequately managed severe migraine in itself may also present a risk to both the mother and child (13).

First, we characterized the MoBa population of migraineurs. Women using migraine pharmacotherapy during pregnancy were more likely to have several known migraine risk factors including poor sleep quality and high pre-gestational body mass index (17). They were also considerably more often on sick leave during pregnancy compared to women without migraine.

Secondly, we wished to investigate the safety of triptans, a widely used medication group against acute migraine attacks. Few previous studies had been conducted on this class of medications, and consequently caution with use in pregnancy is recommended (18). In total, 1045 women in this MoBa sub-study $(1.5 \%)$ used triptans during pregnancy $(12,19)$. In addition, 805 women $(1.1 \%)$ used triptans prior to pregnancy, but not in pregnancy, and were included in the analyses as a disease comparison group. Use of triptans during the first trimester was not associated with any increased risk of major congenital malformations (3.5\% among exposed vs. $2.9 \%$ in the population comparison group, adjusted OR 1.2; 95\% CI 0.7-1.9). Interestingly, women in the migraine comparison group had an increased risk of stillbirth (adjusted OR 5.6; 95\% CI 1.3-23.7). This finding was based on only two exposed cases, and consequently should be explored in yet larger studies.

\section{Medication safety on neurodevelopmental outcomes}

While the majority of medication safety studies in pregnancy focus on immediate pregnancy outcomes, the long-term outcomes, although of equally paramount importance, have received limited attention. The biological plausibility of neurodevelopmental effects of in utero psychotropic exposure is related to their ability to pass the blood-placenta barrier (20) and their ability to bind to receptors in the developing fetus.

In our most recent study published we found that three year old children who had been exposed to paracetamol (=acetaminophen) for more than 28 days during fetal life had poorer gross motor development, communications skills and more externalizing problems compared to their unexposed siblings (21). The effect size was approximately one quarter of a standard deviation (e.g. $\beta$ 0.24) which is of similar magnitude to the negative effect on cognitive and neurodevelopment seen in children exposed to lead in utero $(22,23)$. This is to be considered a substantial effect especially after controlling for shared familial and genetic factors in the sibling design. Moreover, our effect estimates should be reconsidered in light of the public health perspective where a shift in the population central tendency of 0.24 standard deviation will result in a much larger proportion of the population above critical levels of behavioral problems (24) e.g. estimated from $6 \%$ to $10 \%$ for behavioral disorders in our study.

At the same time, results from the Danish National Birth Cohort (DNBC) were published linking prenatal exposure to paracetamol to ADHD in children (25). In this birth cohort, children exposed to paracetamol in utero were more likely to have a hyperkinetic diagnosis (hazard ratio (HR) $1.37 ; 95 \%$ CI $1.19-1.59$ ), to be using ADHD medications (HR 1.29; 95\% CI 1.151.44), or having ADHD-like behaviors at age 7 years (risk ratio $1.13 ; 95 \%$ CI 1.01-1.27). The authors state that the results did not appear to be confounded by potential confounders like maternal inflammation, infection during pregnancy and the mother's mental health problems, and stronger associations were observed with increasing paracetamol exposures.

Because use of paracetamol is frequent in pregnancy, these two Nordic cohort studies have caused concern about neurodevelopmental side effects at the level of the European Medicines Agency (18), and been debated by the scientific community (26-30). Most recently, Damkier et al. question the use of psychometric instruments such as the Ages and Stages Questionnaire (ASQ) to assess infant neurodevelopment (27). In our reply, we point out that we also used other measures in MoBa such as the Child Behaviour Checklist and the Emotionality, Activity and Shyness Temperament Questionnaire (EAS) in addition to a psychomotor milestone (age when the child started walking) (5). In the field of child psychology these are well-know and internationally recognized measurements used clinically to assess child neurodevelopment, but they may be less well-known and accepted by pharmacologists.

Similar findings have recently been published based on data from a New Zealand birth cohort in which prenatal exposure to paracetamol was associated with a significantly higher total difficulty scores (0.25 standard deviation, Strengths and Difficulty Questionnaire) and more symptoms of the DSM-IV hyperactiveimpulsive ADHD subtype at 7 years of age (31). The authors conclude that their findings strengthen the contention that prenatal exposure to paracetamol increases the risk of ADHD-like behaviour in childhood.

Moreover, a Swedish animal study just recently contributed with biological data by showing that exposure to repeated doses of paracetamol $(30+30 \mathrm{mg} / \mathrm{kg}$ bodyweight) in mice induced altered locomotor activity and failure to acquire spatial learning in adulthood with observed changes in key brain regions (32).

$\mathrm{MoBa}$ data have been used in three other studies to assessed long-term neurodevelopmental outcomes after exposure to antidepressants (33) and antiepileptics, respectively $(34,35)$. In the first study, one of the most important findings was the association between longterm in utero exposure to SSRI-antidepressants $(n=161)$ and language delay at three years of age 
(adjusted relative risk ratios 2.30, 95\% CI 1.21-4.37). Symptoms of anxiety and depression in pregnancy, however, were also associated with language delay in offspring, indicating an impact of the underlying maternal psychiatric illness (adjusted relative risk ratios $1.83,95 \%$ CI 1.40-2.40). A recent review of the literature on neurobehavioral outcome of infants exposed in utero concluded that both antidepressant medications and the underlying illness in itself may have negative impact on the developing fetus and that decisions regarding treatment of depression during pregnancy must be made on an individual basis (36).

It is well known that certain antiepileptics may increase the risk of malformations, and some antiepileptics (i.e. valproic acid) may possibly also have an independent negative impact on fetal cognitive development (13). In a first study, a total of $333 \mathrm{MoBa}$ children were exposed to valproate, lamotrigine, carbamazepine, or multiple antiepileptic drugs in utero. At three years of age, the exposed children had increased risk of gross motor delay (7.5\% vs. 3.3\%; OR 2.2, CI 1.1-4.2), poor sentence skills ( $11.2 \%$ vs. $4.8 \%$; OR 2.1 , CI $1.2-$ $3.6)$, and autistic traits $(6.0 \%$ vs. $1.5 \%$; OR 3.4 , CI 1.6-7.0). Confounding by the underlying epileptic illness could not explain the findings as children born to women with epilepsy who did not use antiepileptic drugs had no increased risks and children of fathers with epilepsy generally scored within the normal range (34). In the second study, the impact of breast feeding, while using antiepileptics, on infant neurodevelopment was specifically assessed, but no harmful effects of breastfeeding was detected (35). The authors concluded that women with epilepsy should be encouraged to breastfeed their children irrespective of antiepileptic drug treatment, which is in line with the current literature (13).

\section{Herbal remedies}

In parallel to medication safety studies, we have been working with the use of herbal remedies in pregnancy (37-40). To our surprise, we found that up to $40 \%$ of Norwegian women were using herbal remedies during pregnancy $(38,40)$, despite a lack of data on efficacy and safety in pregnancy for most of these herbs. As the MoBa study includes questions about herbal remedies during pregnancy, this offered an opportunity to study consequences of such use.

In two MoBa sub-studies ( $n=68,522$ women), the safety of the two most frequently used herbals during pregnancy were investigated, namely ginger (41) and cranberry (42). In general, very little information exists on these herbal preparations despite their widespread use.

In the first study, $1,020(1.5 \%)$ women reported using ginger (most commonly against nausea) during pregnancy (41). The use of ginger during pregnancy was not associated with any increased risk of congenital malformation (adjusted OR 0.8, 95\% CI 0.51.4) or with any of the other pregnancy outcomes investigated (i.e. preterm birth and low birth weight, adjusted risk estimates ranging from 0.7 to 1.0$)$ compared to women who had not been exposed (41).

In the second study, 919 (1.3\%) women reported using cranberry during pregnancy, most commonly in relation to urinary tract infections (UTI) (42). No increased risk of major congenital malformations (adjusted OR $0.7,95 \%$ CI $0.4-1.3$ ) or any other negative pregnancy outcomes investigated after use of cranberry were identified in analyses adjusting for confounding factors (adjusted risk estimates ranging from 0.7 to 1.1). Stratified analyses according to use/no use of antibiotics for UTI were performed to investigate whether UTI without conventional therapy could increase the risk of negative pregnancy outcomes. No such effect was found, but study power in these subanalyses were in most cases too low to conclude (42).

\section{Other MoBa studies on medication safety}

Risk of oral clefts after exposure to topical corticosteroids was assessed in one case control study using data from both the MoBa and The Norway Cleft Study (43). No increased risk of oral clefts was detected in either of these populations-based studies.

\section{METHODOLOGICAL CONSIDERATIONS RELEVANT TO MEDICATION SAFETY STUDIES IN MoBA}

Pharmaco-epidemiological studies are vital to our understanding of the safety of medications in pregnancy, but great care must be taken in the analysis and interpretation of observational data. Over the last fifty years, there have been several cases in which widely used drugs were falsely labelled as human teratogens due to signals in pharmaco-epidemiological studies (44), causing women to terminate otherwise-wanted pregnancies, and inducing tremendous levels of anxiety among women and their families. These included the antiemetic Bendectin (pyridoxine/doxylamine) (45) and the antihistamine loratadine (46) to mention a few.

Common methodological challenges in medication safety studies in pregnancy are sample size (e.g., power), errors in measurement of medication use, and bias in the form of selection bias, information bias and confounding. Sources of errors or bias should be acknowledged and discussed, and preferably quantified by performing sensitivity analysis of estimates under a range of assumption about bias direction and magnitude.

The main challenge in several of our MoBa studies focusing on malformations in offspring is that most malformations occur spontaneously at low rates (3$4 \%$ ), and most human teratogens increase such risk modestly. For example, in a cohort study one needs 330 exposed cases and 8000 unexposed controls to show a two-fold increased risk for overall malformations, assuming a baseline prevalence rate of $3 \%$ and $80 \%$ statistical power with an alpha value of 0.05 . 
When dealing with specific malformations, the absolute rates are much lower. For example, cardiac defects occur in approximately $1 \%$ of the population. To exclude a doubling of risk with $80 \%$ statistical power with an alpha value of $0.05,1200$ exposed cases and 8000 controls are necessary. A way to increase the statistical power of relatively small cohort studies of similar design is to synthesize them into a metaanalysis.

The accuracy of medication exposure in MoBa depends on the accuracy of maternal recall. Previous validation studies indicate that completeness of reported medication use in pregnancy depends on the type of medication of interest and on the specificity of the questions asked, with structured questions about indications being more accurate than open-ended questions; i.e., "Have you used any medications in pregnancy?" and "If yes, which?" $(47,48)$. Also, the woman's ability to remember accurately declines as the length of time between the time when the drug was taken and the interview increases. Medications that are taken daily in pregnancy, such as insulin are more accurately reported than short term therapies such as antibiotic eye drops and decongestant nose sprays.

In $\mathrm{MoBa}$, we had the advantage that the women had the opportunity to report medication use for 77 different indications during pregnancy. However, due to the limited space provided in the questionnaires under each indication, underreporting of medication use may occur. This fact, and the low participation rate in $\mathrm{MoBa}$, implies that the prevalence of medication use in pregnancy, most likely, will not be representative for the Norwegian pregnant population at large. Associations between medication exposures and pregnancy outcomes, however, are assumed to be more robust. As $\mathrm{MoBa}$ data is collected prospectively before the pregnancy outcome is known, this would most likely result in a non-differential exposure misclassification and bias of the effect estimates towards 1.0. One potential source of misclassification occurred when several medications were reported and several check boxes for timing of use in pregnancy were crossed out. In such cases, we assumed that these women were exposed to all medications reported in all time periods selected. To determine whether our decision influenced the results of our analyses, we performed sensitivity analyses on different exposure scenarios. In addition, in MoBa, no questions were asked about dosage, and information about the duration of medication use was missing for several women.

A major challenge in observational drug safety studies is the interpretation of causal effects. Often, confounding by indication, socio-economic factors or life style habits cannot be ruled out. Inclusion of a disease comparison group and comparisons of medication effects across indications and across mechanisms of action, are two important approaches that are possible in $\mathrm{MoBa}$, since information about indication, and in some cases, information about severity is included (i.e. symptoms of depression measured by the Symptoms Check List-5 and -8). Similar associations across different indications and among medications with similar modes of action increase the possibility of a causal association.

The possibility that associations are due to genetic or family environmental risks rather than antidepressant use, per se, cannot easily be ruled out in conventional studies. In several of our MoBa studies we are now evaluating the association between prenatal medication exposure and problems in offspring using a sibling control design. The sibling control design may provide one of the most effective approaches to control for family factors when large epidemiological cohorts and sufficient discordant siblings are available (49). This sibling-control design is suitable because siblings share familial environment (e.g. maternal chronic disease) and $50 \%$ of their genetic predisposition, while they may differ on medication exposure during pregnancy.

\section{CRITERIA FOR TERATOGENICITY}

A teratogenic effect could result in several outcomes that are important for establishing the safety of medications in pregnancy. These include congenital malformations, spontaneous abortions, stillbirth, low birth weight, and long-term neurodevelopmental delays and disorders.

The following six criteria are often used to establish whether a medication is teratogenic (50): 1 . The drug should pass the placenta, 2 . There should be a biological plausible explanation for the finding (mechanism of teratogenicity), 3. There should be a relevant temporal relationship between exposure and outcome. The exposure to the medication has to have taken place at a vulnerable time in fetal development (for example, a medication cannot cause a neural tube defect after the neural tube has closed), 4. Specificity is important as most teratogens will produce a certain type of malformation. A specific syndrome increases the likelihood of causation (for example, if anotia (lack of ears) occurs spontaneously in 1:20000 births, and three successive cases of exposure to isotretinoin result in infants born without ears, then this medication is most likely a human teratogen), 5. Replication is important, and should be consistent in at least two or more highquality pharmaco-epidemiological studies, and 6 . Support from animal experiments. Teratogenicity in animals can give important information, but is not essential as results from animal studies cannot automatically be extrapolated to humans. The doses used in animal studies are often ten- to hundredfold in excess of human therapeutic doses.

\section{FUTURE RESEARCH POSSIBILITIES IN MOBA}

There are three especially relevant research possibilities for medication safety studies in MoBa. Firstly, it is becoming increasingly clear to scientists, clinicians and 
the public alike that the reproductive safety of medications cannot be assured without considering potential long-term consequences on the developing brain. Disruptions during fetal neurodevelopment may lead to psychomotor, temperamental and behavioral disabilities. The etiology of many neurodevelopmental disorders, including ADHD, is still poorly understood, but may include exposure to medications in the prenatal period. The nature of $\mathrm{MoBa}$ with a broad range of neurodevelopmental measures and follow-up into adolescence and onward, will allow for these studies, and thus offers to fill an important knowledge gap on longterm medication safety.

Secondly, another recently emerging field in which $\mathrm{MoBa}$ and the biobank can potentially play a leading role is in the field of human pharmaco-epigenetics. Human studies showing alterations in DNA methylation patterns after in utero exposure to medications have just started to emerge, with links to neurobehavioral disorders (51-55). These sparse human findings must be replicated. If proven correct, the consequences for modern medicine will be profound, since it would imply that our current understanding of pharmacology is an oversimplification.

Thirdly, collaboration with other birth cohorts in Europe and North America is promising. Collaboration will not only allow replication of findings, but also the pooling of data to investigate less frequently used medications and rare outcomes that neither of the birth cohorts would have adequate study power to examine alone. Moreover, linkage of $\mathrm{MoBa}$ to other nationwide registries like the Norwegian Prescription Database (NorPD), and to the National Patient Registry will allow for validation of exposure to prescribed medications and inclusion of a medically confirmed diagnosis in addition to self-reported diseases and psychometric measurements, respectively. Advanced statistical techniques will enable us to control complex, intertwined time-varying exposures and confounding factors to obtain estimates of effects that are less biased than traditional regression methods, thereby giving us greater confidence in the validity of our findings.

\section{CONCLUSION}

In practice, pharmacoepidemiological studies provide the best way of evaluating whether an exposure during pregnancy has adverse effects on the developing fetus. The Norwegian Mother and Child Cohort Study has already enabled several medication safety studies in pregnancy, providing reassuring results for antidepressants and supporting current guidelines of avoiding NSAIDs during the first and third trimester. A new signal of behavioral problems after long-term use of paracetamol was detected using a sibling-design. Together with the MoBa biobank, and through linkage to other nationwide registries, the $\mathrm{MoBa}$ study will continue to be an extremely valuable data source for medication safety studies in pregnancy in the future. The knowledge generated from these studies will not only benefit pregnant women in Norway, but women, their children and families worldwide.

\section{ACKNOWLEDGEMENTS}

We thank all our co-authors and all the women who participated in the MoBa study. Four of the studies cited in this article $(10-12,16,17,19)$ are part of the doctorial work of Kateřina Nezvalová-Henriksen entitled "The safety of Antimigraine, Analgesic and Antiinflammatory Drugs during Pregnancy. Results from the Norwegian Mother and Child Cohort Study, the Medical Birth Registry of Norway, and the Norwegian Prescription Database" which she defended at School of Pharmacy, Faculty of Mathematics and Natural Sciences, University of Oslo, Norway in 2013.

\section{REFERENCES}

1. Nordeng H. Drug Utilisation Studies in Pregnancy. In: Drug Utilization Studies. John Wiley \& Sons, 2015.

2. Engeland A, Bramness JG, Daltveit AK, Ronning M, Skurtveit S, Furu K. Prescription drug use among fathers and mothers before and during pregnancy. A population-based cohort study of 106,000 pregnancies in Norway 2004-2006. Br J Clin Pharmacol 2008;65:653-60.

3. Lupattelli A, Spigset O, Twigg MJ, et al. Medication use in pregnancy: a cross-sectional, multinational webbased study. BMJ Open 2014;4:e004365.

4. Reviewer Guidance Evaluating the Risks of Drug Exposure in Human Pregnancies, Food and Drug Administration, 2005.

5. Koren G, Nordeng H. Antidepressant use during pregnancy: the benefit-risk ratio. Am J Obstet Gynecol 2012;207:157-63.

6. Bennett HA, Einarson A, Taddio A, Koren G, Einarson TR. Prevalence of depression during pregnancy: systematic review. Obstet Gynecol 2004;103:698-709.

7. Nordeng H, van Gelder MM, Spigset O, Koren G, Einarson A, Eberhard-Gran M. Pregnancy outcome after exposure to antidepressants and the role of maternal depression: results from the Norwegian Mother and Child Cohort Study. J Clin Psychopharmacol 2012;32:186-94.

8. Lupattelli A, Spigset O, Koren G, Nordeng H. Risk of vaginal bleeding and postpartum hemorrhage after use of antidepressants in pregnancy: a study from the norwegian mother and child cohort study. $J$ Clin Psychopharmacol 2014;34:143-8.

9. van Gelder MM, Roeleveld N, Nordeng H. Exposure to non-steroidal anti-inflammatory drugs during pregnancy and the risk of selected birth defects: a prospective cohort study. PLoS One 2011;6:e22174. 
10. Nezvalova-Henriksen K, Spigset O, Nordeng H. Effects of ibuprofen, diclofenac, naproxen, and piroxicam on the course of pregnancy and pregnancy outcome: a prospective cohort study. BJOG 2013;120:948-59.

11. Nezvalova-Henriksen K, Spigset O, Nordeng H. Effects of codeine on pregnancy outcome: results from a large population-based cohort study. Eur J Clin Pharmacol 2011;67:1253-61.

12. Nezvalova-Henriksen K, Spigset O, Nordeng H. Triptan exposure during pregnancy and the risk of major congenital malformations and adverse pregnancy outcomes: results from the Norwegian Mother and Child Cohort Study. Headache 2010;50:563-75.

13. Schaefer C, Peters PWJ, Miller RK. Drugs during pregnancy and lactation: Treatment options and risk assessment, 2nd edn. Amsterdam: Elsevier, 2007.

14. Ofori B, Oraichi D, Blais L, Rey E, Berard A. Risk of congenital anomalies in pregnant users of non-steroidal anti-inflammatory drugs: A nested case-control study. Birth Defects Res B Dev Reprod Toxicol 2006;77:26879.

15. Briggs GG, Freeman RK, Yaffe SJ. Drugs in Pregnancy and Lactation: a Reference Guide to Fetal and Neonatal Risk, 9th edn. Philadelphia: Wolters Kluwer/Lippincott Williams \& Wilkins, 2011.

16. Nezvalová-Henriksen K, Spigset O, Nordeng H. Erratum to: Effects of codeine on pregnancy outcome: results from a large population-based cohort study. Eur J Clin Pharmacol 2012;68:1689-90.

17. Nezvalova-Henriksen K, Spigset O, Nordeng H. Maternal characteristics and migraine pharmacotherapy during pregnancy: cross-sectional analysis of data from a large cohort study. Cephalalgia 2009;29:1267-76.

18. The Pharmacovigilance Risk Assessment Committee (PRAC) meeting, European Medicines Agency, May 2014.

19. Nezvalova-Henriksen K, Spigset O, Nordeng HM. Errata in "Triptan exposure during pregnancy and the risk of major congenital malformations and adverse pregnancy outcomes: results from the Norwegian Mother and Child Cohort Study". Headache 2012;52:1319-20.

20. Simone C, Derewlany LO, Koren G. Drug transfer across the placenta. Considerations in treatment and research. Clin Perinatol 1994;21:463-81.

21. Brandlistuen RE, Ystrom E, Nulman I, Koren G, Nordeng H. Prenatal paracetamol exposure and child neurodevelopment: a sibling-controlled cohort study. Int J Epidemiol 2013;42:1702-13.

22. Bellinger D, Leviton A, Waternaux C, Needleman H, Rabinowitz M. Longitudinal analyses of prenatal and postnatal lead exposure and early cognitive development. $N$ Engl J Med 1987;316:1037-43.

23. Chiodo LM, Jacobson SW, Jacobson JL. Neurodevelopmental effects of postnatal lead exposure at very low levels. Neurotoxicol Teratol 2004;26:359-71.

24. Bellinger DC. Perspectives on incorporating human neurobehavioral end points in risk assessments. Risk Analysis 2002;22:487-98.

25. Liew Z, Ritz B, Rebordosa C, Lee PC, Olsen J. Acetaminophen use during pregnancy, behavioral problems, and hyperkinetic disorders. JAMA Pediatr 2014;168:313-20.

26. Thiele K, Kessler T, Arck P, Erhardt A, Tiegs G. Acetaminophen and pregnancy: short- and long-term consequences for mother and child. J Reprod Immunol 2013;97:128-39.

27. Damkier P, Pottegard A, Christensen R, Hallas J. Pregnancy and paracetamol: Methodological considerations on the study of associations between in utero exposure to drugs and childhood neurodevelopment. Basic Clin Pharmacol Toxicol 2014, doi: 10.1111/bcpt.12322 [Epub ahead of print].

28. Brandlistuen RE, Ystrom E, Nulman I, Koren G, Nordeng H. Authors' Response: More research on paracetamol is required. Int J Epidemiol 2014;43:975-6.

29. Cannell JJ. Paracetamol, oxidative stress, vitamin D and autism spectrum disorders. Int J Epidemiol 2014; 43:974-5.

30. Brandlistuen RE, Ystrom E, Nulman I, Koren G, Nordeng H. Response to "Pregnancy and Paracetamol: Methodological Considerations on the Study of Associations between In Utero Exposure to Drugs and Childhood Neurodevelopment". Basic Clin Pharmacol Toxicol 2014, doi: 10.1111/bcpt.12339.

31. Thompson JM, Waldie KE, Wall CR, Murphy R, Mitchell EA. Associations between acetaminophen use during pregnancy and ADHD symptoms measured at ages 7 and 11 years. PLoS One 2014;9:e108210.

32. Viberg H, Eriksson P, Gordh T, Fredriksson A. Paracetamol (acetaminophen) administration during neonatal brain development affects cognitive function and alters its analgesic and anxiolytic response in adult male mice. Toxicol Sci 2014;138:139-47.

33. Skurtveit S, Selmer R, Roth C, Hernandez-Diaz S, Handal M. Prenatal exposure to antidepressants and language competence at age three: results from a large population-based pregnancy cohort in Norway. BJOG 2014, doi: 10.1111/1471-0528.12821 [Epub ahead of print].

34. Veiby G, Daltveit AK, Schjolberg S, et al. Exposure to antiepileptic drugs in utero and child development: a prospective population-based study. Epilepsia 2013;54:1462-72. 
35. Veiby G, Engelsen BA, Gilhus NE. Early child development and exposure to antiepileptic drugs prenatally and through breastfeeding: a prospective cohort study on children of women with epilepsy. JAMA Neurol 2013;70:1367-74.

36. Suri R, Lin AS, Cohen LS, Altshuler LL. Acute and long-term behavioral outcome of infants and children exposed in utero to either maternal depression or antidepressants: a review of the literature. J Clin Psychiatry 2014;75:e1142-52.

37. Kennedy DA, Lupattelli A, Koren G, Nordeng H. Herbal medicine use in pregnancy: results of a multinational study. BMC Complement Altern Med 2013;13:355.

38. Nordeng H, Bayne K, Havnen GC, Paulsen BS. Use of herbal drugs during pregnancy among 600 Norwegian women in relation to concurrent use of conventional drugs and pregnancy outcome. Complement Ther Clin Pract 2011;17:147-51.

39. Holst L, Nordeng H, Haavik S. Use of herbal drugs during early pregnancy in relation to maternal characteristics and pregnancy outcome. Pharmacoepidemiol Drug Saf 2008;17:151-9.

40. Nordeng H, Havnen GC. Use of herbal drugs in pregnancy: a survey among 400 Norwegian women. Pharmacoepidemiol Drug Saf 2004;13:371-80.

41. Heitmann K, Nordeng H, Holst L. Safety of ginger use in pregnancy: results from a large population-based cohort study. Eur J Clin Pharmacol 2013;69:269-77.

42. Heitmann K, Nordeng H, Holst L. Pregnancy outcome after use of cranberry in pregnancy - the Norwegian Mother and Child Cohort Study. BMC Complement Altern Med 2013;13:345.

43. Skuladottir H, Wilcox A, McConnaughey R, Vindenes H, Lie RT. First-trimester nonsystemic corticosteroid use and the risk of oral clefts in Norway. Ann Epidemiol 2014;24:635-40.

44. Schwarz EB, Moretti ME, Nayak S, Koren G. Risk of hypospadias in offspring of women using loratadine during pregnancy: a systematic review and meta-analysis. Drug Saf 2008;31:775-88.

45. Koren G. Medication safety in pregnancy and breastfeeding: the evidence-based A-to-Z clinician's pocket guide. New York: McGraw-Hill Medical, 2007.

46. Kallen B, Olausson PO. No increased risk of infant hypospadias after maternal use of loratadine in early pregnancy. Int J Med Sci 2006;3:106-7.

47. de Jong-van den Berg LT, Waardenburg CM, Haaijer-Ruskamp FM, Dukes MN, Wesseling H. Drug use in pregnancy: a comparative appraisal of data collecting methods. Eur J Clin Pharmacol 1993;45:9-14.

48. Mitchell AA, Cottler LB, Shapiro S. Effect of questionnaire design on recall of drug exposure in pregnancy. Am J Epidemiol 1986;123:670-6.

49. Susser E, Eide MG, Begg M. Invited commentary: The use of sibship studies to detect familial confounding. Am J Epidemiol 2010;172:537-9.

50. Shepard TH. "Proof" of human teratogenicity. Teratology 1994;50:97-8.

51. Soubry A, Murphy S, Huang Z, et al. The effects of depression and use of antidepressive medicines during pregnancy on the methylation status of the IGF2 imprinted control regions in the offspring. Clin Epigenetics 2011;3:2.

52. Oberlander TF, Weinberg J, Papsdorf M, Grunau R, Misri S, Devlin AM. Prenatal exposure to maternal depression, neonatal methylation of human glucocorticoid receptor gene (NR3C1) and infant cortisol stress responses. Epigenetics 2008;3:97-106.

53. Conradt E, Lester BM, Appleton AA, Armstrong DA, Marsit CJ. The roles of DNA methylation of NR3C1 and 11beta-HSD2 and exposure to maternal mood disorder in utero on newborn neurobehavior. Epigenetics 2013;8:1321-9.

54. Non AL, Binder AM, Kubzansky LD, Michels KB. Genome-wide DNA methylation in neonates exposed to maternal depression, anxiety, or SSRI medication during pregnancy. Epigenetics 2014;9:964-72.

55. van Mil NH, Steegers-Theunissen RP, Bouwland-Both MI, et al. DNA methylation profiles at birth and child ADHD symptoms. J Psychiatr Res 2014;49:51-9. 